\title{
Phonon superradiance and phonon laser effect in nanomagnets
}

\author{
E. M. Chudnovsky ${ }^{1}$ and D. A. Garanin ${ }^{1,2}$ \\ ${ }^{1}$ Department of Physics and Astronomy, Lehman College, City University of New York, \\ 250 Bedford Park Boulevard West, Bronx, New York 10468-1589, U.S.A. \\ ${ }^{2}$ Institut für Physik, Johannes-Gutenberg-Universität, D-55099 Mainz, Germany
}

(Dated: May 18, 2018)

\begin{abstract}
We show that the theory of spin-phonon processes in paramagnetic solids must take into account the coherent generation of phonons by the magnetic centers. This effect should drastically enhance spin-phonon rates in nanoscale paramagnets and in crystals of molecular nanomagnets.

PACS numbers: $75.50 . \mathrm{Xx}, 76.30 .-\mathrm{v}, 43.35 .+\mathrm{d}$
\end{abstract}

The problem of spin-lattice interactions is almost as old as quantum theory of solids [1]. It is about computing the amplitude of the transition between spin states of a magnetic atom due to the spontaneous emission of a phonon or due to, e.g., the Raman scattering of an existing thermal phonon. These processes are responsible for the width of the paramagnetic resonance and for other spin-lattice relaxation effects. In this Letter we shall demonstrate that all previous works on the subject have missed the collective nature of the relaxation when the wavelength of the phonon is comparable to the distance between the magnetic atoms. General theoretical treatment of this effect has become possible due to the recently established mechanism of the dynamic spinphonon coupling [2],

$$
\widehat{H}_{s-p h}=-\hbar \mathbf{S} \cdot \boldsymbol{\Omega}, \quad \boldsymbol{\Omega}(\mathbf{r})=\frac{1}{2} \nabla \times \dot{\mathbf{u}}(\mathbf{r}),
$$

where $\mathbf{S}$ is the spin of the atom, $\boldsymbol{\Omega}$ is the angular velocity of the local rotation of the crystal due to a transverse phonon, and $\mathbf{u}$ is the phonon displacement field. Eq. (1) has the following origin. The crystal field (i.e., the magnetic anisotropy) that determines spin states of an atom in a solid is defined in a local coordinate frame coupled to the crystal axes. In that coordinate frame the local rotation of the lattice is equivalent to the magnetic field, $\mathbf{B}=\hbar \boldsymbol{\Omega} / g \mu_{B}$ (with $g$ being the gyromagnetic factor for the spin), that has Zeeman interaction with $\mathbf{S}$. Since the local rotations of the crystal lattice by phonons are small, all matrix elements computed in the rotating frame and in the laboratory frame are practically identical. The beauty of this approach is that it has no unknown parameters. For, e.g., spontaneous emission of a phonon, all parameters of the crystal field enter the interaction through the phonon frequency only. The latter is determined by the distance between the spin levels and can be directly measured, leaving no fitting parameters for comparison between theory and experiment. Another way to look at this interaction is through the conservation of the total angular momentum, spin + lattice. In order to conserve the angular momentum, the transition between the spin states of an atom produces a local elastic twist that propagates out as a transverse phonon. This Letter is based upon observation that an assembly of the magnetic atoms in the excited state must interact via transverse phonons very much the same as atoms inter- act via photons in the laser physics. The deep analogy between the two problems can be seen by noticing from Eq. (11) that $\dot{\mathbf{u}} / 2 g \mu_{B}$ is equivalent to the vector potential of the electromagnetic field. The huge $\left(c / v_{t}\right)^{3}$ ratio of the phonon density of states to the photon density of states (with $v_{t}$ being the speed of the transverse sound) makes such a phonon laser much more powerful than the photon laser based upon magnetic dipoles.

We consider two nearly degenerate states of spin $\mathbf{S}$, described by the Hamiltonian for pseudospin 1/2,

$$
\widehat{H}_{0}=-\frac{1}{2} \hbar \boldsymbol{\omega}_{0} \cdot \boldsymbol{\sigma}, \quad \hbar \boldsymbol{\omega}_{0}=W \mathbf{e}_{z}+\Delta \mathbf{e}_{x}
$$

where $\boldsymbol{\sigma}$ is the Pauli matrix, $\Delta$ is the splitting of the levels at the avoided crossing, and $W=E_{-1}-E_{1}$ is the energy difference for the two degenerate states at $\Delta=0$ ( \pm 1 denoting spin up and down). We label these states as $\left|\psi_{-1}\right\rangle$ and $\left|\psi_{1}\right\rangle$, then $E_{ \pm 1}=\left\langle\psi_{ \pm 1}\left|\widehat{H}_{\mathbf{S}}\right| \psi_{ \pm 1}\right\rangle$ and $\Delta=$ $2\left\langle\psi_{-1}\left|\widehat{H}_{\mathbf{S}}\right| \psi_{1}\right\rangle$, where $\widehat{H}_{\mathbf{S}}$ is the crystal-field Hamiltonian for the spin $\mathbf{S}$. Choosing the new $z$ axis for pseudospins in the direction of $\boldsymbol{\omega}_{0}$, one obtains the eigenvalues and the eigenfunctions of $\widehat{H}_{0}: E_{ \pm}= \pm(1 / 2) \sqrt{W^{2}+\Delta^{2}}$ and $\left|\psi_{ \pm}\right\rangle=\left(C_{\mp}\left|\psi_{1}\right\rangle \pm C_{ \pm}\left|\psi_{-1}\right\rangle\right) / \sqrt{2}$ with $C_{ \pm}=$ $\sqrt{1 \pm W / \sqrt{\Delta^{2}+W^{2}}}$, whereas $\hbar \boldsymbol{\omega}_{0}=\sqrt{W^{2}+\Delta^{2}} \mathbf{e}_{z}$. The projection of the spin-phonon interaction, Eq. (1), onto the two-state basis is

$$
\widehat{H}_{s-p h}=-\frac{\hbar}{2} \boldsymbol{\sigma} \cdot \overleftrightarrow{\mathbf{g}} \cdot(\nabla \times \dot{\mathbf{u}}) \equiv-\frac{\hbar}{2} \sigma_{\alpha} g_{\alpha \beta}(\nabla \times \dot{\mathbf{u}})_{\beta} .
$$

For tunneling between up and down states of spin $\mathbf{S}$, $\left|\psi_{ \pm 1}\right\rangle=| \pm S\rangle$ and

$$
\begin{aligned}
& \mathbf{g}_{x}=\mathbf{e}_{z} S C_{+} C_{-}=\mathbf{e}_{z} S \frac{\Delta}{\sqrt{W^{2}+\Delta^{2}}}, \quad \mathbf{g}_{y}=\mathbf{0} \\
& \mathbf{g}_{z}=-\mathbf{e}_{z} S \frac{1}{2}\left(C_{+}^{2}-C_{-}^{2}\right)=-\mathbf{e}_{z} S \frac{W}{\sqrt{W^{2}+\Delta^{2}}} .
\end{aligned}
$$

For many two-state particles coupled to phonons the effective many-body Hamiltonian is

$$
\widehat{H}=-\frac{\hbar}{2} \sum_{i} \boldsymbol{\omega}_{0} \cdot \boldsymbol{\sigma}_{i}-\frac{\hbar}{2} \sum_{i} \boldsymbol{\sigma}_{i} \cdot \overleftrightarrow{\mathrm{g}} \cdot\left[\nabla \times \dot{\mathbf{u}}\left(\mathbf{r}_{i}\right)\right]+\widehat{H}_{p h}
$$


where $\widehat{H}_{p h}$ is the Hamiltonian of non-interacting phonons. We use canonical quantization of phonons,

$$
\mathbf{u}=\sqrt{\frac{\hbar}{2 M N}} \sum_{\mathbf{k} \lambda} \frac{\mathbf{e}_{\mathbf{k} \lambda} e^{i \mathbf{k} \cdot \mathbf{r}}}{\sqrt{\omega_{k \lambda}}}\left(a_{\mathbf{k} \lambda}+a_{-\mathbf{k} \lambda}^{\dagger}\right),
$$

where $M$ is the mass of the unit cell, $N$ is the number of cells, $\mathbf{e}_{\mathbf{k} \lambda}$ are unit polarization vectors, $\lambda=t, t, l$ denotes polarization, and $\omega_{k \lambda}=v_{\lambda} k$ is the phonon frequency. The spin-phonon Hamiltonian then becomes

$$
\widehat{H}_{s-p h}=-\frac{\hbar}{2} \sum_{\mathbf{k} \lambda} \sum_{i} e^{i \mathbf{k} \cdot \mathbf{r}_{i}} \boldsymbol{\sigma}_{i} \cdot \mathbf{G}_{\mathbf{k} \lambda}\left(a_{\mathbf{k} \lambda}-a_{-\mathbf{k} \lambda}^{\dagger}\right),
$$

where $\mathbf{G}_{\mathbf{k} \lambda}$ is due to transverse phonons only, $\lambda=t, t$,

$$
\mathbf{G}_{\mathbf{k} \lambda} \equiv \sqrt{\frac{\hbar \omega_{k \lambda}}{2 M N}} \overleftrightarrow{\mathbf{g}} \cdot\left[\mathbf{k} \times \mathbf{e}_{\mathbf{k} \lambda}\right], \quad \mathbf{G}_{\mathbf{k} \lambda}=-\mathbf{G}_{-\mathbf{k} \lambda}
$$

In the Heisenberg representation for time-dependent operators, $i \hbar[d \widehat{O}(t) / d t]=[\widehat{O}(t), \widehat{H}]$, one obtains, with account of the commutation relations, $\left[\sigma_{i \alpha}, \sigma_{j \beta}\right]=$ $2 i \epsilon_{\alpha \beta \gamma} \sigma_{i \gamma} \delta_{i j}$ and $\left[a_{\mathbf{k} \lambda}, a_{\mathbf{k}^{\prime} \lambda^{\prime}}^{\dagger}\right]=\delta_{\mathbf{k k}^{\prime}} \delta_{\lambda \lambda^{\prime}}$, the following coupled equations:

$$
\begin{array}{r}
\dot{\boldsymbol{\sigma}}_{i}=\boldsymbol{\sigma}_{i} \times\left[\boldsymbol{\omega}_{0}+\sum_{\mathbf{k} \lambda} e^{i \mathbf{k} \cdot \mathbf{r}_{i}} \mathbf{G}_{\mathbf{k} \lambda}\left(a_{\mathbf{k} \lambda}-a_{-\mathbf{k} \lambda}^{\dagger}\right)\right] \\
\dot{a}_{\mathbf{k} \lambda}=-\left(i \omega_{k \lambda}+\gamma_{k \lambda}\right) a_{\mathbf{k} \lambda}+\frac{i}{2} \sum_{i} e^{-i \mathbf{k} \cdot \mathbf{r}_{i}} \boldsymbol{\sigma}_{i} \cdot \mathbf{G}_{\mathbf{k} \lambda} .
\end{array}
$$

Due to the linear interaction between $\boldsymbol{\sigma}_{i}$ and $\mathbf{u}$, Eq. (5) conserves $\left(\sum_{i} \boldsymbol{\sigma}_{i}\right)^{2}$ for uniform rotations. Consequently, $\boldsymbol{\sigma}_{i}$ in Eqs. (9) and (10) can be treated as a semiclassical average over distances large compared to the spacing between magnetic atoms but small compared to the phonon wavelength. We are interested in the interaction of such local polarization with a semiclassical phonon field. One can write the Heisenberg operators as

$$
\sigma_{i+}(t)=\tilde{\sigma}_{i+}(t) e^{-i \omega_{0} t}, \quad a_{\mathbf{k} \lambda}(t)=\tilde{a}_{\mathbf{k} \lambda}(t) e^{-i \omega_{k \lambda} t},
$$

where $\tilde{\sigma}_{i+}(t)$ and $\tilde{a}_{\mathbf{k} \lambda}(t)$ are slow variables. Keeping only resonant terms in Eqs. (9) and (10), and neglecting the hybridization of phonon and pseudospin modes, one obtains after integration on time

$$
a_{\mathbf{k} \lambda}=\frac{1}{4} \frac{\sum_{i} e^{-i \mathbf{k} \cdot \mathbf{r}_{i}} \sigma_{i+} G_{\mathbf{k} \lambda,-}}{\omega_{k \lambda}-\omega_{0}-i \gamma_{k \lambda}}
$$

where $G_{\mathbf{k} \lambda, \pm}=G_{\mathbf{k} \lambda, x} \pm i G_{\mathbf{k} \lambda, y}$. The insertion of $a_{\mathbf{k} \lambda}$ and $a_{-\mathbf{k} \lambda}^{\dagger}$ into Eq. (9) then gives the system of equations:

$$
\begin{aligned}
& \dot{\sigma}_{i \pm}=\mp i \omega_{0} \sigma_{i \pm}-\frac{1}{2} \sigma_{i z} \sum_{j} \Gamma_{i j} \sigma_{j \pm} \\
& \dot{\sigma}_{i z}=\frac{1}{4} \sum_{j} \Gamma_{i j}\left(\sigma_{j+} \sigma_{i-}+\sigma_{j-} \sigma_{i+}\right)
\end{aligned}
$$

with

$$
\Gamma_{i j}=\frac{1}{N} \sum_{\mathbf{k}} e^{i \mathbf{k} \cdot\left(\mathbf{r}_{i}-\mathbf{r}_{j}\right)} V_{\mathbf{k}}^{2} \frac{2 \gamma_{k}}{\left(\omega_{k}-\omega_{0}\right)^{2}+\gamma_{k}^{2}}
$$

and

$$
V_{\mathbf{k}}^{2} \equiv \frac{N}{4} \sum_{\lambda=t, t}\left(G_{\mathbf{k} \lambda, x}^{2}+G_{\mathbf{k} \lambda, y}^{2}\right) .
$$

For a small sample of size, $L \lesssim \lambda_{0} \sim v_{t} / \omega_{0}$, containing $\mathcal{N} \gg 1$ magnetic atoms or molecules $(1 \ll \mathcal{N} \ll N)$ that are initially polarized in one direction, one can use $e^{i \mathbf{k} \cdot\left(\mathbf{r}_{i}-\mathbf{r}_{j}\right)} \cong 1$ in Eq. (14). Eqs. (13) then preserve the length of the total polarization, $\sum_{j} \boldsymbol{\sigma}_{j}$. The unit vector $\boldsymbol{\sigma}=(1 / \mathcal{N})\left(\sum_{j} \boldsymbol{\sigma}_{j}\right)$ satisfies the Landau-Lifhitz equation,

$$
\dot{\boldsymbol{\sigma}}=\left[\boldsymbol{\sigma} \times \boldsymbol{\omega}_{0}\right]-\alpha\left[\boldsymbol{\sigma} \times\left[\boldsymbol{\sigma} \times \boldsymbol{\omega}_{0}\right]\right],
$$

where $\alpha=\mathcal{N} \Gamma_{1} /\left(2 \omega_{0}\right)$ is the dimensionless damping constant and $\Gamma_{1}=N^{-1} \sum_{\mathbf{k}} V_{\mathbf{k}}^{2} 2 \pi \delta\left(\omega_{k}-\omega_{0}\right)$ is the one-atom spin-phonon rate. Replacing $N^{-1} \sum_{\mathrm{k}}$.. by $v_{0} \int d^{3} k /(2 \pi)^{3} \ldots\left(v_{0}\right.$ being the unit cell volume) and writing $V_{\mathbf{k}}^{2}$ with the help of Eqs. (4), (8), and (15) as

$$
V_{\mathbf{k}}^{2}=\frac{S^{2}}{8 \hbar} \frac{\Delta^{2}}{M v_{t}^{2}} \frac{\omega_{k}^{3}}{\omega_{0}^{2}}\left(1-\frac{k_{z}^{2}}{k^{2}}\right),
$$

one obtains for the one-atom spin-phonon rate [2]:

$$
\Gamma_{1}=\frac{S^{2}}{12 \pi \hbar} \frac{\Delta^{2}}{M v_{t}^{2}} \frac{\omega_{0}^{3}}{\omega_{D}^{3}}=\frac{S^{2}}{12 \pi \hbar} \frac{\Delta^{2} \omega_{0}^{3}}{\rho v_{t}^{5}},
$$

where $\omega_{D} \equiv v_{t} / v_{0}^{1 / 3}$ is the Debye frequency for the tranverse phonons and $\rho=M / v_{0}$ is the mass density of the crystal. It is easy to check that $\Gamma_{\mathrm{SR}}=\mathcal{N} \Gamma_{1}$ is the rate of the relaxation of $\sigma_{z}$ in Eq. (16), that is, the longitudinal relaxation rate. The transverse relaxation rate for $\sigma_{ \pm}$is $\Gamma_{\mathrm{SR}} / 2$. This is Dicke superradiance [3] with the rate exceeding the one-atom spin-phonon rate by a large factor $\mathcal{N}$. Eq. (16), among other situations, describes the case when all pseudospins are initially prepared in the excited state, $\sigma_{z}=-1$, and then relax collectively towards the ground state, $\sigma_{z}=1$, by emitting coherent phonons of frequency $\omega_{0}$. Because $V_{\mathbf{k}}^{2}$ of Eq. (17) is proportional to $\sin ^{2} \theta=1-k_{z}^{2} / k^{2}$, the angular distrubution of the emitted phonons is maximal in the direction perpendicular to the quantization axis $z$.

For large samples, $\lambda \lesssim L$, the phase of the emitted phonons is no longer constant throughout the sample, and one should also study inhomogeneous solutions of Eqs. (13). For that purpose it is convenient to switch to the Fourier harmonics $\sigma_{i \pm}=\mathcal{N}^{-1} \sum_{\mathbf{q}} e^{ \pm i \mathbf{q} \cdot \mathbf{r}_{i}} \sigma_{\mathbf{q} \pm}$ and $\sigma_{i z}=\mathcal{N}^{-1} \sum_{\mathbf{q}} e^{i \mathbf{q} \cdot \mathbf{r}_{i}} \sigma_{\mathbf{q} z}$. When the paramagnetic body is a small part of a large solid matrix, the phonon modes are practically continuous in the $\mathbf{k}$-space while $\mathbf{q}$ is quantized. The first of Eqs. (13) then becomes

$$
\dot{\sigma}_{\mathbf{q} \pm}=\mp i \omega_{0} \sigma_{\mathbf{q} \pm}-\frac{1}{2} \sum_{\mathbf{q}^{\prime \prime}} \Gamma\left(\mathbf{q}^{\prime \prime}\right) \sigma_{\left[ \pm\left(\mathbf{q}-\mathbf{q}^{\prime \prime}\right)\right], z} \sigma_{\mathbf{q}^{\prime \prime} \pm}
$$

where

$$
\Gamma(\mathbf{q})=\frac{1}{N} \sum_{\mathbf{k}} V_{\mathbf{k}}^{2}\left|F_{\mathbf{k}-\mathbf{q}}\right|^{2} \frac{\gamma_{k}}{\left(\omega_{k}-\omega_{0}\right)^{2}+\gamma_{k}^{2}},
$$


and $F_{\mathbf{k}}=\mathcal{N}^{-1} \sum_{i} e^{i \mathbf{k} \cdot \mathbf{r}_{i}} \cong V^{-1} \int d \mathbf{r} e^{i \mathbf{k} \cdot \mathbf{r}}$. For a rectangular body of dimensions $L_{\alpha}(\alpha=x, y, z)$ it gives

$$
F_{\mathbf{k}}=\prod_{\alpha} \frac{\sin \left(k_{\alpha} L_{\alpha} / 2\right)}{k_{\alpha} L_{\alpha} / 2}
$$

whereas for a sphere of radius $R$ one obtains $F_{\mathbf{k}}=f(x) \equiv$ $3 x^{-3}(\sin x-x \cos x)$ with $x=k R$.

Superradiance corresponds to the $\mathbf{q}=0$ mode in Eq. (19). For $\gamma_{\mathbf{k}} \rightarrow 0$ it occurs at a rate

$$
\begin{aligned}
\Gamma_{\mathrm{SR}} & =\mathcal{N} v_{0} \int \frac{d^{3} k}{(2 \pi)^{3}} V_{\mathbf{k}}^{2}\left|F_{\mathbf{k}}\right|^{2} 2 \pi \delta\left(\omega_{k}-\omega_{0}\right) \\
& =\mathcal{N} \Gamma_{1}\left\langle V_{\mathbf{k}_{0}}^{2}\left|F_{\mathbf{k}_{0}}\right|^{2}\right\rangle /\left\langle V_{\mathbf{k}_{0}}^{2}\right\rangle
\end{aligned}
$$

where $\langle\ldots\rangle$ denotes the average over the directions of $\mathbf{k}_{0}$ that satisfy $\omega_{k_{0}}=\omega_{0}$. For $k_{\alpha} L_{\alpha} \ll 1$ one can replace $F_{\mathbf{k}_{0}}$ by 1 , and the second of Eqs. (22) reduces to the Dicke rate, $\Gamma_{\mathrm{SR}}=\mathcal{N} \Gamma_{1}$. In the general case, the enhancement of the relaxation rate due to superradiance is given by

$$
\frac{\Gamma_{\mathrm{SR}}}{\Gamma_{1}}=\frac{n_{M}}{k_{0}^{3}} \Upsilon, \quad n_{M}=\frac{\mathcal{N}}{V} .
$$

where $n_{M}$ is concentration of magnetic atoms and

$$
\Upsilon=k_{0}^{3} V\left\langle V_{\mathbf{k}_{0}}^{2}\left|F_{\mathbf{k}_{0}}\right|^{2}\right\rangle /\left\langle V_{\mathbf{k}_{0}}^{2}\right\rangle
$$

depends on the wavelength and on the shape of the magnetic body. Since the maximal value of $\Upsilon$ is of order 1 , the maximal enhancement is always given by the dimensionless ratio $n_{M} / k_{0}^{3}$. For a sphere one obtains $\Upsilon=(4 \pi / 3)\left(k_{0} R\right)^{3} f^{2}\left(k_{0} R\right)$. The destructive interference, $\Upsilon=0$, occurs at $k_{0} R \cong \pi n$, where $n$ is an integer. At $k_{0} R \gg 1, \Upsilon\left(k_{0} R\right) \sim 1 /\left(k_{0} R\right)$. That is, $\Gamma_{\mathrm{SR}}$ goes down to $\Gamma_{1}$ only at $k_{0} R \sim n_{M} / k_{0}^{3}$, that can be very large. For a box of dimensions $L_{\alpha}=b_{\alpha} L$, the geometrical factor $\Upsilon$ must be computed numerically. For a cube of size $L, \Upsilon\left(k_{0} L\right)$ is qualitatively similar to that of the sphere. It almost vanishes at $k_{0} L=2 \pi n$, which corresponds to the destructive interference of phonons emitted perpendicular to the cube faces. The choice of $L_{x} \neq L_{y}$ causes a more complicated pattern shown in Fig. 1h.

An interesting observation is that the increase of the $z$ dimension of the body beyond the wavelength of the radiation does not lead to the decrease of the superradiant rate. The latter is the consequence of the suppression of phonons emitted in that direction, Eq. (17). For a sample of a prism shape with $k_{0} L_{x} \sim k_{0} L_{y} \ll 1$ and arbitrary $k_{0} L_{z}$, one obtains $\Upsilon=\left(k_{0} L_{x}\right)\left(k_{0} L_{y}\right) \tilde{\Upsilon}\left(k_{0} L_{z}\right)$, where $\tilde{\Upsilon}(u) \cong u$ at $u \equiv k_{0} L_{z} \ll 1$ and $\tilde{\Upsilon}(u) \cong 3 \pi / 2$ at $u \gg 1$, see Fig. 10. Another interesting case is a flat sample with $k_{0} L_{x} \ll 1, \quad b_{y} \sim b_{z} \sim 1$ and arbitrary $u=k_{0} L$. In this case one can write $\Upsilon=\left(k_{0} L_{x}\right) \tilde{\Upsilon}\left(u, b_{y}, b_{z}\right)$, where $\tilde{\Upsilon} \cong u^{2} b_{y} b_{z}$ at $u \ll 1$ and $\tilde{\Upsilon} \cong 3 \pi$ at $u \gg 1$, Fig. 10. The latter is a thin-film limit for which $\Upsilon$ depends neither on $b_{y, z}$ nor even on the shape of the film area. The dependence of $\Upsilon$ on the thickness of such $y z$-film is

$$
\Upsilon=6 \pi \frac{1-\cos \left(k_{0} L_{x}\right)}{k_{0} L_{x}} .
$$
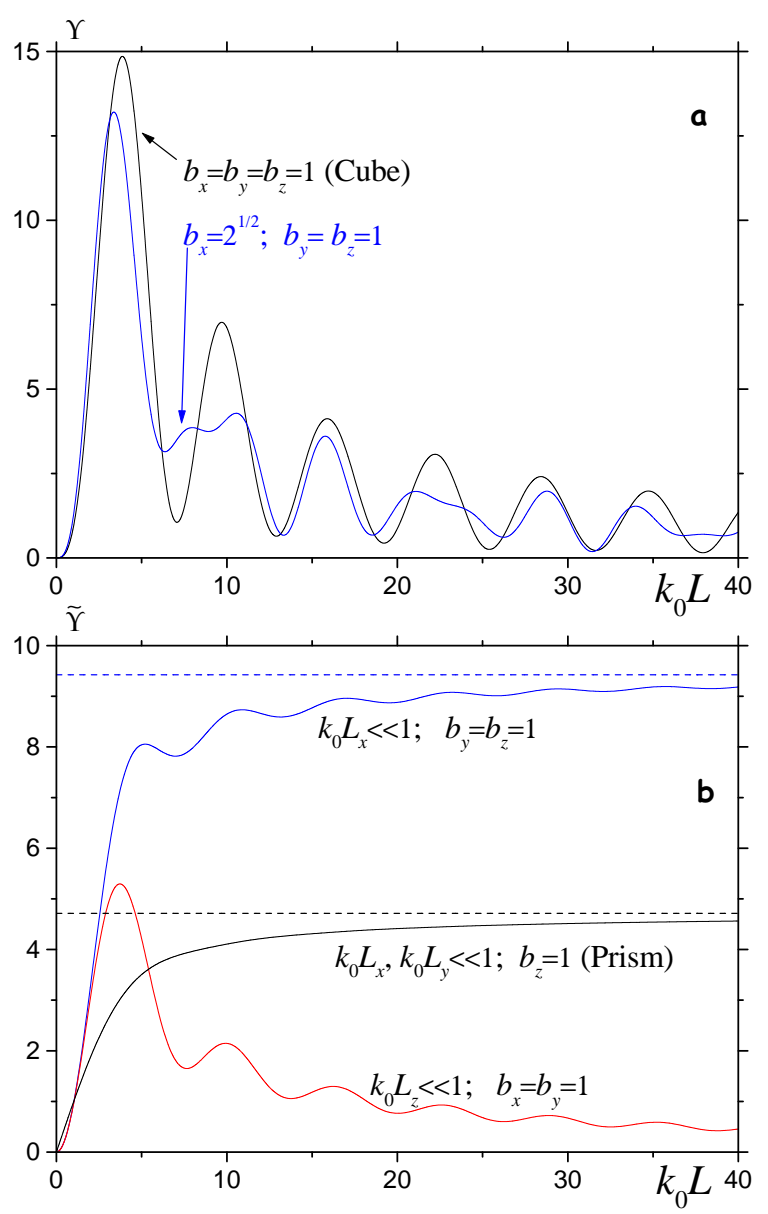

FIG. 1: $a-\Upsilon$ for rectangular geometry; $b-\tilde{\Upsilon}$ for the film and prism geometries. $L_{\alpha}=b_{\alpha} L, \alpha=x, y, z$.

In this geometry the phonons are emitted in the direction $x$ perpendicular to the film. The emission rate can be as large as $\Gamma_{\mathrm{SR}, \max } \approx 13.7\left(n_{M} / k_{0}^{3}\right) \Gamma_{1}$ at $k_{0} L_{x} \approx 2.33$, while the intensity of sound is proportional to the film surface. One should notice the difference from the case of the $x y$-film, Fig. 1b, for which the superradiant rate decreases with increasing the dimensions of the film surface beyond the phonon wavelength.

Let us now consider the relaxation of the non-zero $\sigma_{\mathbf{q}}$ harmonics in macroscopic samples for a particular case of the maximal inversion, $\sigma_{z} \cong-1$. At $k_{0} L \gg 1$, because of the factor $\left|F_{\mathbf{k}-\mathbf{q}}\right|^{2}$ in Eq. (20), the phonon momenta $\mathbf{k}$ that contribute to Eq. (20) must be very close to $\mathbf{q}$. Under the above conditions, Eq. (19) simplifies to

$$
\dot{\sigma}_{\mathbf{q} \pm}=\mp i \omega_{0} \sigma_{\mathbf{q} \pm}+\frac{1}{2} \Gamma(\mathbf{q}) \sigma_{\mathbf{q} \pm}
$$

where $\Gamma(\mathbf{q})$ is now the increment rate given by

$$
\Gamma(\mathbf{q})=V_{\mathbf{q}}^{2} \frac{2 \gamma_{q}}{\left(\omega_{q}-\omega_{0}\right)^{2}+\gamma_{q}^{2}} .
$$

The highest rate corresponds not to $\mathbf{q}=\mathbf{0}$, as for the superradiance, but to $\mathbf{q}$ that provides the minimal value of 
$\omega_{q}-\omega_{0}$ determined by the previously neglected hybridization of the spin and phonon modes, $(\Delta \omega)_{\min } \sim V_{\mathbf{k}_{0}}$. This is the laser mode that grows at a rate

$$
\Gamma_{L}=\frac{V_{\mathbf{k}_{\mathbf{0}}, \max }^{2} \gamma_{k_{0}}}{V_{\mathbf{k}_{\mathbf{0}}, \max }^{2}+\gamma_{k_{0}}^{2}}, \quad V_{\mathbf{k}_{\mathbf{0}}, \max }^{2}=\frac{S^{2}}{8 \hbar} \frac{\Delta^{2} \omega_{0}}{M v_{t}^{2}},
$$

where $V_{\mathbf{k}_{\mathbf{0}} \text {, max }}^{2}$ is the maximal value of $V_{\mathbf{k}_{\mathbf{0}}}^{2}$ achieved for $\mathbf{k}$ perpendicular to the $z$ axis. The maximal value of $\Gamma_{L}$ on $\gamma_{k_{0}}$ is $\Gamma_{L, \max }=V_{\mathbf{k}_{\mathbf{0}}, \max }$, and the enhancement of the spin-phonon relaxation is very large:

$$
\frac{\Gamma_{L, \max }}{\Gamma_{1}}=\frac{3 \sqrt{2}}{S}\left(\frac{\omega_{D}}{\omega_{0}}\right)^{3}\left(\frac{\hbar \omega_{0}}{\Delta}\right)^{1 / 2}\left(\frac{M v_{t}^{2}}{\Delta}\right)^{1 / 2} .
$$

At $V_{\mathbf{k}_{\mathbf{0}}, \max } \ll \gamma_{k_{0}}$, Eq. (28) reduces to the well-known laser formula, $\Gamma_{L}=V_{\mathbf{k}_{\mathbf{0}} \text {, max }}^{2} / \gamma_{k_{0}}$. As in optical lasers, the increment rate $\Gamma_{L}$ must compete with the dephasing rate $\Gamma_{2}$ due to, e.g., inhomogeneous broadening of $\Delta$. The laser generation requires $V_{\mathbf{k}_{0}, \max }^{2}>\gamma_{k_{0}} \Gamma_{2}$. It will be the preferred decay mode of the inverted population of spin states if $\omega_{0}=\sqrt{W^{2}+\Delta^{2}} / \hbar$ coincides with one of the frequencies of the quantized phonon modes of the crystal. For a small crystal, this condition can be satisfied only at certain values of $W$ and $\Delta$. This can be achieved by continuously changing longitudinal or transverse magnetic field. Since the distance between these resonant field values is inversely proportional to the size of the body, the resonance condition, at a finite $\gamma_{k_{0}}$, must be satisfied at any field for a body of size $L \gtrsim v_{t} / \gamma_{k_{0}}$.

Our results on phonon superradiance alter the existing theory of EPR in nanomagnets at low temperature. They show that one cannot properly account for the width of the EPR by simply computing one-phonon processes for isolated magnetic atoms. When the wavelength of the phonons, $\lambda_{0}=2 \pi / k_{0}$, is greater than the distance between magnetic atoms, the collective processes come into play, greatly enhancing the rates. Observation of EPR lines requires $k_{B} T \lesssim \hbar \omega_{0}$, which at $T \lesssim 1 \mathrm{~K}$ translates into $\lambda_{0} \lesssim 50 \mathrm{~nm}$. Thus, in the kelvin temperature range, our results on superradiance are applicable to nanomagnets of size below $50 \mathrm{~nm}$ and to thin films of thickness less than $50 \mathrm{~nm}$. Larger samples should exhibit superradiance at lower temperatures. The direct study of phonon superradiance requires inverted population of spin states. In conventional paramagnets this can be achieved in a pulsed magnetic field. In microscrystals of molecular nanomagnets the inverted population of spin states is easy to achieve even in a slowly varying field [4]. Note that in crystals of small size the phonon superradiance always wins over the electromagnetic superradiance [5].

The existence of the laser phonon mode may be consistent with the experimental evidence [6, 7, 8, 9] that the low-temperature spin-phonon rates in molecular nanomagnets are few orders of magnitude higher than the computed one-molecule spin-phonon rates [10, 11]. In molecular magnets the two-level system is formed every time the external magnetic field, $B$, produces a resonance between the states described by the magnetic quantum numbers $m$ and $m^{\prime}$. For the $\left(m, m^{\prime}\right)$ resonance, $S$ in all our formulas must be replaced by $\left|m-m^{\prime}\right| / 2$, while $W=$ $\left|m-m^{\prime}\right| g \mu_{B} B$. Consider, e.g., a typical field-sweep experiment in $\mathrm{Fe}_{8}$, when the field crosses the $(10,-8)$ resonance and is $0.1 \mathrm{~T}$ past the resonance. Taking $\Delta \simeq 10^{-4} \mathrm{~K}$ [12], $M \simeq 5 \times 10^{-21} \mathrm{~g}$, and $v_{t} \simeq 10^{5} \mathrm{~cm} / \mathrm{s}$, one obtains: $\omega_{0} \simeq 10^{11} \mathrm{~s}^{-1}, \quad \Gamma_{1} \simeq 10^{-5} \mathrm{~s}^{-1}$, but $\Gamma_{L, \max } \simeq 10^{4} \mathrm{~s}^{-1}$, which would provide the $m=10 \Rightarrow m^{\prime}=-8$ spinphonon relaxation in less than 1-ms time. This may explain spin-phonon avalanches observed in sufficiently large crystals of molecular magnets [13, 14]. The laser rate $\Gamma_{L}=V_{\mathbf{k}_{\mathbf{0}} \text {, max }}^{2} / \gamma_{k_{0}}$ has a distinctive $\omega_{0}$ dependence that should be seen in experiment. At low temperature $\gamma_{k_{0}} \propto \omega_{0}^{4}$ due to the scattering of phonons by the inhomogeneities of the crystal [15], while $V_{\mathbf{k}_{\mathbf{0}}, \max }^{2} \propto \omega_{0}$. Consequently, $\Gamma_{L} \propto \omega_{0}^{-3}$, which is opposite to the $\omega_{0}$ dependence of the one-molecule spin-phonon rate, $\Gamma_{1} \propto \omega_{0}^{3}$.

This work has been supported by the NSF Grant No. EIA-0310517.
[1] See, e.g., A. Abragam and B. Bleaney, Electron Paramagnetic Resonance of Transition Ions (Clarendon Press, Oxford, 1970) and references therein.

[2] E. M. Chudnovsky, Phys. Rev. Lett. 72, 3433 (1994); E. M. Chudnovsky and X. Martinez-Hidalgo, Phys. Rev. B66, 054412 (2002); E. M. Chudnovsky, Phys. Rev. Lett. 92, 120405 (2004).

[3] R. H. Dicke, Phys. Rev. 9399 (1954).

[4] R. Sessoli, D. Gatteschi, A. Caneschi, and M. A. Novak, Nature (London) 365, 141 (1993).

[5] E. M. Chudnovsky and D. A. Garanin Phys. Rev. Lett. 89, 157201 (2002).

[6] J. R. Friedman, M. P. Sarachik, J. Tejada, and R. Ziolo, Phys. Rev. Lett. 76, 3830 (1996).

[7] J. R. Friedman, M. P. Sarachik, and R. Ziolo, Phys. Rev. B58, R14729 (1998).

[8] Kyungwha Park, M. A. Novotny, N. S. Dalal, S. Hill, and P. A. Rikvold, J. Appl. Phys. 91, 7167 (2002).
[9] M. Dressel, B. Gorshunov, K. Rajagopal, S. Vontragool, and A. A. Mukhin, Phys. Rev. B67, 060405 (2003).

[10] J. Villain, F. Hartmann-Boutron, R. Sessoli, and A. Rettori, Europhys. Lett. 27, 159 (1994).

[11] D. A. Garanin and E. M. Chudnovsky, Phys. Rev. B56, 11102 (1997).

[12] W. Wernsdorfer and R. Sessoli, Science 284, 133 (1999).

[13] C. Paulsen and J.-G. Park, pp. 189-207 in Quantum Tunneling of Magnetization - QTM'94, Edited by L. Gunther and B. Barbara (Kluwer, Dordrecht, Netherlands, 1995).

[14] E. del Barco, J. M. Hernandez, M. Sales, J. Tejada, H. Rakoto, J. M. Broto, and E. M. Chudnovsky, Phys. Rev. B60, 11898 (1999).

[15] See, e.g., D. A. Garanin, Ann. Phys. (N.Y.) 218, 293 (1992), and references therein. 